\title{
Optimal bladder filling during high-dose-rate intracavitary brachytherapy for cervical cancer: a dosimetric study
}

\author{
Umesh Mahantshetty, DMRT, MD, DNBR', Saurabha Shetty, MD', Dipanjan Majumder, MD', Pranjal Adurkar, DRP², \\ Jamema Swamidas, DRP, PhD², Reena Engineer, MD, DNB', Supriya Chopra, MD, DNB!' \\ Shyamkishore Shrivastava, MD, DNB' \\ 'Department of Radiation Oncology, Tata Memorial Hospital, ${ }^{2}$ Department of Medical Physics, Tata Memorial Hospital, Parel, Mumbai, India
}

\begin{abstract}
Purpose: The aim of this study is to compare 3D dose volume histogram (DVH) parameters of bladder and other organs at risk with different bladder filling protocol during high-dose-rate intracavitary brachytherapy (HDR-ICBT) in cervical cancer, and to find optimized bladder volume.

Material and methods: This dosimetric study was completed with 21 patients who underwent HDR-ICBT with computed tomography/magnetic resonance compatible applicator as a routine treatment. Computed tomography planning was done for each patient with bladder emptied (series 1), after $50 \mathrm{ml}$ (series 2), and $100 \mathrm{ml}$ (series 3) bladder filling with a saline infusion through the bladder catheter. Contouring was done on the Eclipse Planning System. 7 Gy to point A was prescribed with the standard loading patterns. Various 3D DVH parameters including $0.1 \mathrm{cc}, 1 \mathrm{cc}$, $2 \mathrm{cc}$ doses and mean doses to the OAR's were noted. Paired $t$-test was performed.

Results: The mean $( \pm$ SD) bladder volume was $64.5( \pm 25)$ cc, $116.2( \pm 28)$ cc, and $172.9( \pm 29)$ cc, for series 1,2 , and 3, respectively. The $0.1 \mathrm{~cm}^{3}, 1 \mathrm{~cm}^{3}, 2 \mathrm{~cm}^{3}$ mean bladder doses for series 1 , series 2 , and series 3 were $9.28 \pm 2.27 \mathrm{~Gy}$, $7.38 \pm 1.72 \mathrm{~Gy}, 6.58 \pm 1.58 \mathrm{~Gy} ; 9.39 \pm 2.28 \mathrm{~Gy}, 7.85 \pm 1.85 \mathrm{~Gy}, 7.05 \pm 1.59 \mathrm{~Gy}$, and $10.09 \pm 2.46 \mathrm{~Gy}, 8.33 \pm 1.75 \mathrm{~Gy}$, $7.6 \pm 1.55 \mathrm{~Gy}$, respectively. However, there was a trend towards higher bladder doses in series 3 . Similarly, for small bowel dose $0.1 \mathrm{~cm}^{3}, 1 \mathrm{~cm}^{3}$, and $2 \mathrm{~cm}^{3}$ in series 1, 2, and 3 were $5.44 \pm 2.2 \mathrm{~Gy}, 4.41 \pm 1.84 \mathrm{~Gy}, 4 \pm 1.69 \mathrm{~Gy} ; 4.57 \pm 2.89 \mathrm{~Gy}$, $3.78 \pm 2.21 \mathrm{~Gy}, 3.35 \pm 2.02 \mathrm{~Gy}$, and $4.09 \pm 2.38 \mathrm{~Gy}, 3.26 \pm 1.8 \mathrm{~Gy}, 3.05 \pm 1.58 \mathrm{~Gy}$. Significant increase in small bowel dose in empty bladder (series 1) compared to full bladder (series 3) $(p=0.03)$ was noted. However, the rectal and sigmoid doses were not significantly affected with either series.

Conclusions: Bladder filling protocol with $50 \mathrm{ml}$ and $100 \mathrm{ml}$ was well tolerated and achieved a reasonably reproducible bladder volume during cervical brachytherapy. In our analysis so far, there is no significant impact of bladder filling on DVH parameters, although larger bladders tend to have higher doses. Small bowel doses are lesser with higher bladder volumes. Further evaluation and validation are necessary.

Key words: cervical cancer, cervical carcinoma, dosimetry, intracavitary brachytherapy.

\section{Purpose}

Intracavitary brachytherapy (ICBT) forms the foundation of treatment for cervical cancer. The International Commission on Radiation Units and Measurements (ICRU) Report 38 in 1985, have given ICRU definition to define and report reference bladder and rectal point doses during ICBT on conventional radiography [1]. In recent past, use of newer imaging modalities like computed tomography $(\mathrm{CT})$, magnetic resonance imaging (MRI), and image-based treatment planning has permitted for better evaluation in terms of dose-volume parameters rather than ICRU point doses. This has allowed a better understanding of the dose volume histogram (DVH) effects on the organs at risk (OAR's), mainly rectum, bladder, and sigmoid. Various studies have shown that the ICRU points do not represent the actual doses to these normal surrounding structures, especially bladder $[2,3,4]$. Furthermore, variation in bladder and rectal filling status during fractionated brachytherapy planning additionally complicates these issues.

Various bladder filling protocols during ICBT have been suggested, with no clear consensus. The bladder filling protocols vary from emptying with indwelling
Address for correspondence: Prof. Umesh Mahantshetty, DMRT, MD, DNBR, Department of Radiation Oncology, Tata Memorial Hospital, 1123, 11 ${ }^{\text {th }}$ floor, Homi Bhabha Building, Tata Memorial Hospital, Parel, 400012 Mumbai, India, phone: +91 22 24177168, 凶e-mail: drumeshm@gmail.com
Received: 10.06 .2016 Accepted: 12.04 .2017 Published: 28.04.2017 
urinary catheter (ICRU 38 recommendations) to a known limited filling status (e.g., $50 \mathrm{cc}$ ) to full bladder. In order to understand the effect of bladder filling on 3D DVH parameters and to determine an optimum bladder volume during high-dose-rate intracavitary brachytherapy (HDR-ICBT) in cervical cancer we undertook this dosimetric study.

\section{Material and methods}

Twenty-one patients with histologically proven cervical cancer clinical FIGO (International Federation of Gynecology and Obstetrics) stage IIB-IIIB were included in this study after obtaining a written informed consent. These patients received external beam radiotherapy (EBRT) with conventional open field portals to a dose of 50 Gy in 25 fractions along with weekly concurrent cisplatin $\left(40 \mathrm{mg} / \mathrm{m}^{2}\right)$ chemotherapy. This was followed by 2-3 fractions (7 Gy each once weekly) of HDR-ICBT. All these patients underwent HDR-ICBT with CT compatible ring applicators. Following the first ICBT application, three series of CT scans were acquired for each individual patient for planning. The CT scans were taken from the lumbosacral junction to the ischial tuberosity with slice thickness of $3 \mathrm{~mm}$ for all the three series. The first series (series 1 , empty bladder) was taken after completely emptying the bladder with a mild negative suction under aseptic conditions using aseptic syringe. Two additional CT scan series were taken after infusion of saline by $50 \mathrm{ml}$ (series 2) and $50 \mathrm{ml}$ more to make it $100 \mathrm{ml}$ (series 3) through the indwelling bladder catheter. These series of empty (series 1), $50 \mathrm{ml}$ (series 2), and $100 \mathrm{ml}$ (series 3) bladder capacity CT scans were transferred to the planning system. Bladder, rectum, sigmoid colon, and small bowel were contoured on all the three series of CT scans for each individual patient. The bladder was contoured from bladder neck to superiorly as visible. The rectum was contoured from the recto-sigmoid junction to the bottom of the ischial tuberosity. The sigmoid colon was contoured from the recto-sigmoid junction to the level of the fundus of the uterus. The small bowel was defined as the peritoneal cavity containing bowel, excluding the sigmoid colon, rectum, and bladder in the pelvis. Treatment planning was performed on all the three series for each patient on the Eclipse Planning System (version 8.1; Varian Medical Systems, Inc., Palo Alto, CA, USA). Dwell positions were determined for each series according to the ring size and tandem length. Treatment planning was done with $7 \mathrm{~Gy}$ to point A prescription with the standard loading patterns for the tandem-ring configuration in all the three CT scan series for each patient. Since this is a dosimetric study, no optimization was allowed after normalization of doses to point A. ICRU bladder point doses and DVH for bladder, rectum, and small bowel were obtained. The 3D DVH parameters for bladder, rectum, sigmoid, and small bowel were evaluated, including $0.1 \mathrm{~cm}^{3}, 1 \mathrm{~cm}^{3}$, and $2 \mathrm{~cm}^{3}$ doses as per GYN GEC-ESTRO (Groupe Européen de Curiethérapie European Society for Radiotherapy and Oncology) recommendations. The OAR's doses were compiled for empty bladder (series 1 ), $50 \mathrm{ml}$ (series 2), and $100 \mathrm{ml}$ filling (series 3). Cor- relation between ICRU bladder point and $2 \mathrm{~cm}^{3}$ bladder doses was also studied. The spatial location of bladder $2 \mathrm{~cm}^{3}$ volumes for all the 3 series were evaluated further.

In order to objectively assess the spatial location of bladder $2 \mathrm{~cm}^{3}$ volumes with different bladder filling status, the methodology implemented was to track the center of volume (COV). This was done by contouring the bladder $2 \mathrm{~cm}^{3}$ volumes in all the 3 series CT data sets. The empty bladder CT data set as fixed; $50 \mathrm{ml}$ and $100 \mathrm{ml}$ bladder CT data sets were co-registered to the fixed CT by aligning to the applicators. Following registration, the bladder $2 \mathrm{~cm}^{3}$ volumes from $50 \mathrm{ml}$ and $100 \mathrm{ml} \mathrm{CT}$ data sets were copied into the fixed CT data set. The overlap volumes between the different series were noted. Further, an isocentre was assigned at the COV on the Treatment Planning System (TPS) eclipse. This aspect was utilized for obtaining the COV coordinates for each of the three structures namely, bladder 2cc_empty, bladder 2cc_50 ml, and bladder 2cc_100 ml. Three temporary external beam plans were created, where in the structure of interest was made the COV (target volume). The COV X (lateral), Y (longitudinal), and $Z$ (vertical) co-ordinates were recorded.

In order to find the relative displacement of the $2 \mathrm{cc}$ volume, the COV of the bladder 2cc_empty was set at origin. The COV co-ordinates for the bladder 2cc_50 ml and bladder 2cc_100 ml volumes were plotted with respect to the bladder 2cc_empty COV at origin for each patient. The displacement in the anterior-posterior, superior-inferior, and lateral directions were plotted per patient for the bladder 2cc_50 $\mathrm{ml}$ and $100 \mathrm{ml}$ COV with respect to the bladder 2cc_empty COV at origin. A 3D graph was generated with all the COV points mapped (Figure 1).

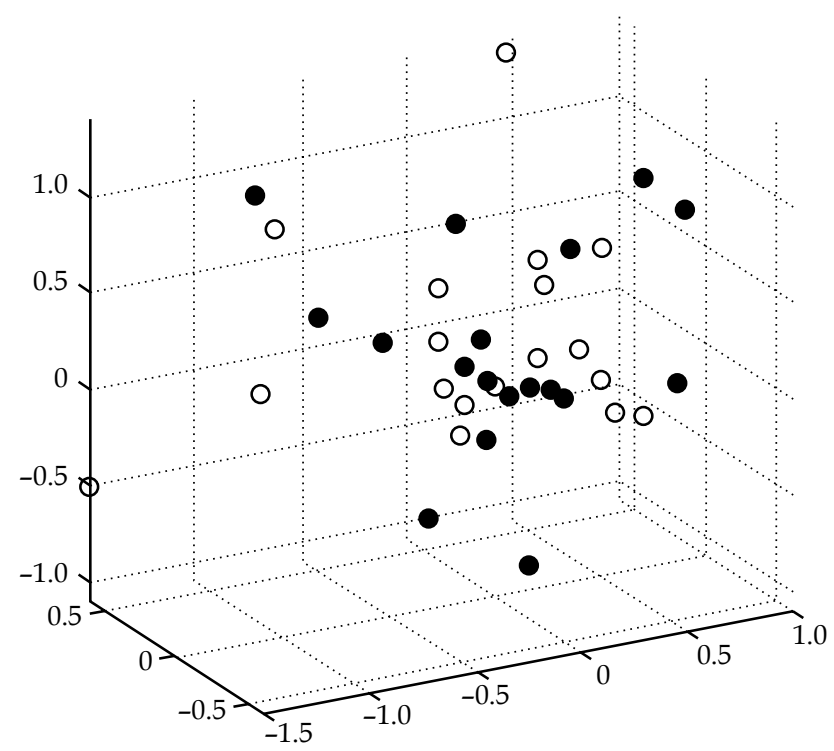

- COM of bladder 2cc_100 ml o COM of bladder 2cc_50 ml

Fig. 1. 3D graph of the center of volume/center of mass (COV/COM) of the bladder dose volumes based on bladder filling, with respect to empty bladder location 


\section{Statistical analysis}

The bladder volume in the three different CT series was noted. The volumes of the different structure sets and the doses received in each series were entered. Descriptive statistics was used for computing mean, median, range, and standard deviations. Paired $t$-test was performed using SPSS 21 software.

\section{Results}

Twenty-one patients underwent CT planning during their first HDR-ICBT planning with empty bladder, $50 \mathrm{ml}$, and $100 \mathrm{ml}$ bladder filling. Data sets and various dose parameters were available for final analysis.

\section{Impact of bladder filling on bladder doses}

There was no significant difference in mean $( \pm$ SD) ICRU bladder point dose $3.8 \pm 1.95 \mathrm{~Gy}, 3.64 \pm 1.86 \mathrm{~Gy}$, and $3.81 \pm 1.7 \mathrm{~Gy}$. The mean ( $\pm \mathrm{SD}$ ) bladder volume was 64.5 $( \pm 25) \mathrm{cc}, 116.2( \pm 28) \mathrm{cc}$, and $173( \pm 29) \mathrm{cc}$, for series 1, 2, and 3, respectively. The $0.1 \mathrm{~cm}^{3}, 1 \mathrm{~cm}^{3}$, and $2 \mathrm{~cm}^{3}$ mean ( \pm SD) bladder doses for series 1 were $9.28 \pm 2.27 \mathrm{~Gy}$, $7.38 \pm 1.72 \mathrm{~Gy}, 6.58 \pm 1.58 \mathrm{~Gy}$, for series 2 and series 3 they were $9.39 \pm 2.28 \mathrm{~Gy}, 7.85 \pm 1.85 \mathrm{~Gy}, 7.05 \pm 1.59 \mathrm{~Gy}$, and $10.09 \pm 2.46 \mathrm{~Gy}, 8.33 \pm 1.75 \mathrm{~Gy}, 7.6 \pm 1.55 \mathrm{~Gy}$, respectively. The differences between the ICRU bladder point and bladder $2 \mathrm{~cm}^{3}$ doses across all the 3 series was highly significant $(p=0.000)$. The $1 \mathrm{~cm}^{3}$ and $2 \mathrm{~cm}^{3}$ bladder doses are approximately 2 times higher than the ICRU bladder points for series 2 and 3 (Table 1). However, the bladder dose for all $0.1 \mathrm{~cm}^{3}, 1 \mathrm{~cm}^{3}$, and $2 \mathrm{~cm}^{3}$ were slightly greater in full bladder rather than empty bladder, which was statistically significant $(p=0.029)$.

\section{Impact of bladder filling on other OAR structures}

The mean rectal volume (37 cc, $38 \mathrm{cc}$, and $42 \mathrm{cc}$ ), sigmoid volumes (55 cc, $58 \mathrm{cc}$, and $61 \mathrm{cc}$ ), and small bowel volume (111 cc, $84 \mathrm{cc}$, and $95 \mathrm{cc}$ ) among the three series were compared. The $0.1 \mathrm{~cm}^{3}$ mean small bowel dose for series 1,2 , and 3 were $5.44 \pm 2.2 \mathrm{~Gy}, 4.57 \pm 2.89 \mathrm{~Gy}$, and $4.09 \pm 2.38 \mathrm{~Gy}$, respectively. The $1 \mathrm{~cm}^{3}$ mean $( \pm \mathrm{SD})$ small bowel doses for series 1,2 , and 3 were $4.41 \pm 1.84 \mathrm{~Gy}$, $3.78 \pm 2.21 \mathrm{~Gy}$, and $3.26 \pm 1.8 \mathrm{~Gy}$, respectively. The $2 \mathrm{~cm}^{3}$ small bowel dose in series 1,2 , and 3 were $4 \pm 1.69 \mathrm{~Gy}$, $3.35 \pm 2.02 \mathrm{~Gy}$, and $3.05 \pm 1.58 \mathrm{~Gy}$, respectively. There was a significant increase in the small bowel doses in se- ries 1 (empty) as compared to series $3(p=0.03)$, while there was a trend towards higher doses in series 2 as compared to series $3(p=0.055)$ and no significant difference between series 1 and 2 over small bowel $0.1 \mathrm{~cm}^{3}$, $1 \mathrm{~cm}^{3}$, and $2 \mathrm{~cm}^{3}$ doses. The $0.1 \mathrm{~cm}^{3}, 1 \mathrm{~cm}^{3}$, and $2 \mathrm{~cm}^{3} \mathrm{sig}$ moid dose for series 1 were $8.06 \pm 2.04 \mathrm{~Gy}, 6.56 \pm 1.52 \mathrm{~Gy}$, $5.88 \pm 1.28 \mathrm{~Gy}$, respectively; for series 2 , the values were $7.99 \pm 2.02 \mathrm{~Gy}, 6.6 \pm 1.48 \mathrm{~Gy}, 6 \pm 1.27 \mathrm{~Gy}$, respectively; for series 3 , the values were $8 \pm 2.29 \mathrm{~Gy}, 6.52 \pm 1.62 \mathrm{~Gy}, 5.92 \pm$ $1.42 \mathrm{~Gy}$, respectively. For rectum, the $0.1 \mathrm{~cm}^{3}, 1 \mathrm{~cm}^{3}$, and $2 \mathrm{~cm}^{3}$ dose in series 1 were $7.04 \pm 3.22 \mathrm{~Gy}, 5.65 \pm 2.69 \mathrm{~Gy}$, $5.06 \pm 2.43 \mathrm{~Gy}$, respectively; for series 2 , those values were $6.52 \pm 2.96 \mathrm{~Gy}, 5.33 \pm 2.46 \mathrm{~Gy}, 4.79 \pm 2.2 \mathrm{~Gy}$, respectively; and for series 3 , the values were $6.53 \pm 2.7 \mathrm{~Gy}, 5.34 \pm$ $2.3 \mathrm{~Gy}, 4.82 \pm 2.07 \mathrm{~Gy}$, respectively. The rectal and sigmoid doses not differ significantly among the different series.

\section{Spatial distribution (surface dose rendering) of 2 cc bladder volumes}

The mean $( \pm S D)$ overlap between the three series was $0.1 \mathrm{cc}( \pm 0.1)$. The overlap between series $1 \& 2$, series 2 \& 3 , and series $3 \& 1$ were $0.3 \mathrm{cc}( \pm 0.2), 0.4 \mathrm{cc}( \pm 0.3), 0.3 \mathrm{cc}$ $( \pm 0.2)$, respectively. The COV of bladder 2 cc volume for the $50 \mathrm{ml}$ and $100 \mathrm{ml}$ series shifted within $5 \mathrm{~mm}$ radius sphere with respect to its position for bladder empty series (Figure 1).

On bladder filling with $50 \mathrm{ml}$, the average displacement of the COV 2 cc bladder dose volume in the lateral, anterior-posterior, and superior-inferior directions were $2.5 \mathrm{~mm}( \pm 4.7),-0.6 \mathrm{~mm}( \pm 5.4)$, and $0.2 \mathrm{~mm}( \pm 2.4)$, respectively. Similarly, on bladder filling with $100 \mathrm{ml}$, the average displacement in the lateral, antero-posterior, superior - inferior directions were $1 \mathrm{~mm}( \pm 2.6), 1.3 \mathrm{~mm}$ $( \pm 6.3)$, and $1.1 \mathrm{~mm}( \pm 4.7)$, respectively. Figure 2 shows the displacement in the lateral, anterior-posterior, and superior-inferior directions that were plotted per patient for the bladder 2cc_50 ml and bladder 2cc_100 ml COV with respect to the bladder 2cc_empty COV at origin. The relative displacement of OARs with different filling protocol are shown in Figure 3.

\section{Discussion}

Image based HDR brachytherapy in cervical cancer is evolving with promising results [5]. Various parameters such as bladder filling, rectal filling, movements of sigmoid colon, and variation in vaginal packing have an impact on dosimetry and intra/inter fraction varia-

Table 1. Comparison of ICRU (International Commission of Radiation Units and Measurements) bladder point dose to volume dose

\begin{tabular}{|c|c|c|c|c|c|c|}
\hline $\begin{array}{l}\text { Bladder } \\
\text { filling }\end{array}$ & $\begin{array}{c}\text { ICRU point } \\
\text { Mean dose Gy } \\
( \pm S D)\end{array}$ & $\begin{array}{c}\mathrm{D}_{0.1 \mathrm{cc}} \\
\text { Mean dose Gy } \\
( \pm \mathrm{SD})\end{array}$ & $\begin{array}{c}D_{1 c c} \\
\text { Mean dose Gy } \\
( \pm S D)\end{array}$ & $\begin{array}{c}\mathrm{D}_{2 \mathrm{cc}} \\
\text { Mean dose Gy } \\
( \pm \mathrm{SD})\end{array}$ & $\begin{array}{c}1 \text { cc mean dose/ } \\
\text { ICRU point mean } \\
\text { dose }\end{array}$ & $\begin{array}{l}2 \text { cc mean dose/ } \\
\text { ICRU point mean } \\
\text { dose }\end{array}$ \\
\hline Empty & $3.80 \pm 1.95$ & $9.28 \pm 2.27$ & $7.38 \pm 1.72$ & $6.58 \pm 1.58$ & 1.94 & 1.73 \\
\hline Partially full & $3.64 \pm 1.86$ & $9.39 \pm 2.28$ & $7.85 \pm 1.85$ & $7.05 \pm 1.59$ & 2.15 & 1.93 \\
\hline Full & $3.81 \pm 1.70$ & $10.09 \pm 2.46$ & $8.33 \pm 1.75$ & $7.60 \pm 1.55$ & 2.19 & 2.00 \\
\hline
\end{tabular}

ICRU - International Commission of Radiation Units and Measurements; $D_{0.1 c c} D_{1 c c} D_{2 c c}$-minimum dose to the most exposed $0.1 \mathrm{~cm}^{3}, 1 \mathrm{~cm}^{3}, 2 \mathrm{~cm}^{3}$ 


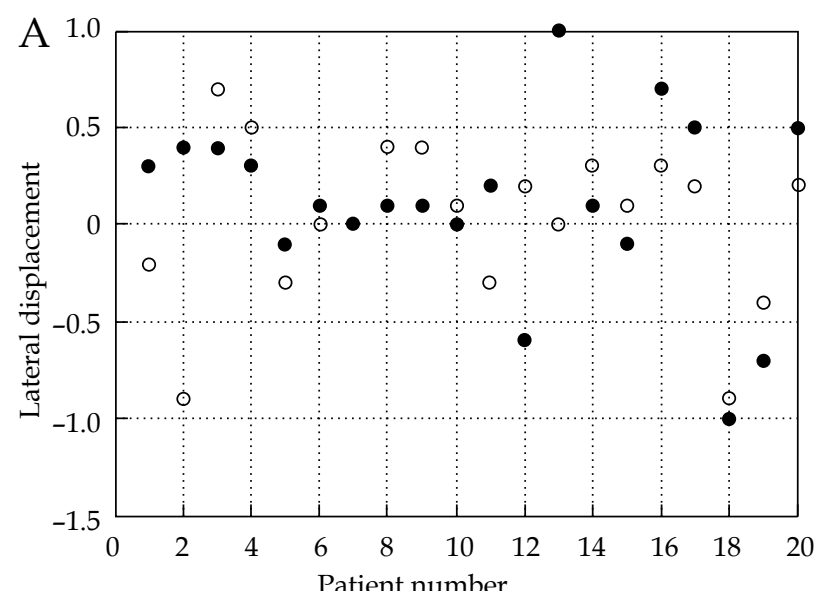

- COM of bladder 2cc_100 ml o COM of bladder 2cc_50 ml

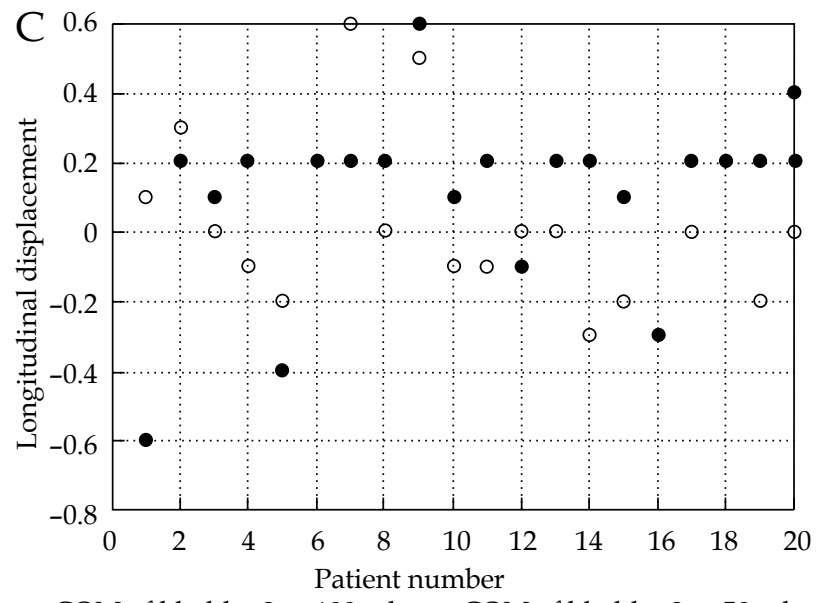

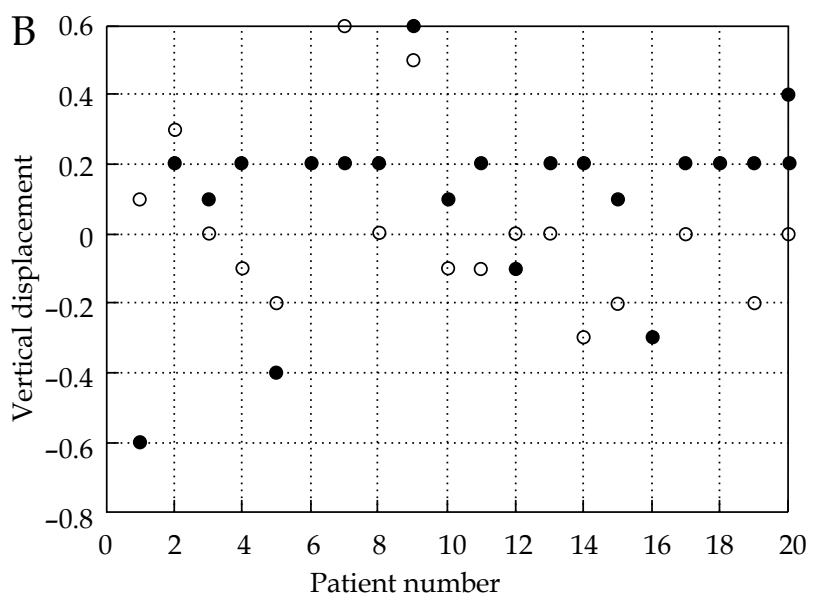

- COM of bladder 2cc_100 ml o COM of bladder 2cc_50 ml

Fig. 2. A) Lateral displacement of the bladder dose volumes based on bladder filling. B) Vertical displacement of the bladder dose volumes based on bladder filling. C) Longitudinal displacement of the bladder dose volumes based on bladder filling

- COM of bladder 2cc_100 ml o COM of bladder 2cc_50 ml
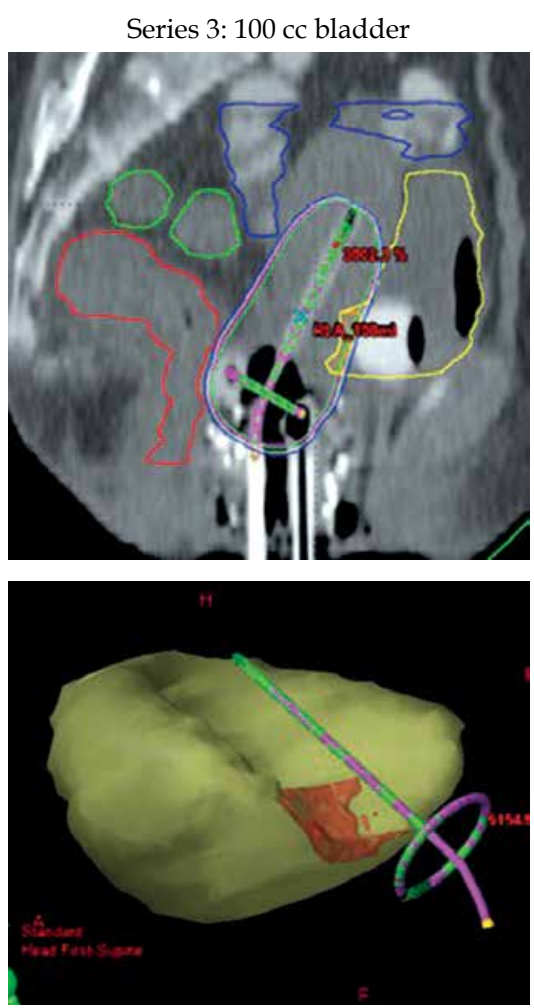

Fig. 3. Dosimetry of organs at risk at different bladder filling protocol 
tions [6]. In this study, we investigated the dosimetric impact of bladder filling status during HDR intracavitary cervical brachytherapy. Although different practice patterns of full or empty bladder have been reported, the ICRU 38 recommends an empty bladder with indwelling Foley's catheter while others with limited bladder filling or full bladder during treatment delivery without any consensus [7]. The highlights of our study are presented below.

\section{Reasonably constant and reproducible bladder volumes during brachytherapy}

Although empty bladder was ensured with continuous negative suction, the empty bladder volume still had some residual urine with a contoured volume of $64 \mathrm{cc}$ ( $\pm 25 \mathrm{cc}$ ). Further, a reproducible bladder volumes (limited filling and full bladder) was achieved after instillation of $50 \mathrm{ml}$ and $100 \mathrm{ml}$ sterile water through indwelling catheter with mean bladder volumes of 116 ( $\pm 28 \mathrm{cc})$ and $173 \mathrm{cc}( \pm 29 \mathrm{cc})$, respectively. Our study demonstrates a reasonably constant bladder volumes that could be achieved with the bladder filling protocol utilized.

\section{Bladder filling and impact on the bladder doses during brachytherapy}

In our study, there was no significant impact on the ICRU bladder point doses with bladder filling protocols. This may be due to the fact that the Foley's bulb is always ensured to be against the bladder base with mild traction. Studies show that doses to the ICRU bladder point do not correlate well with bladder complications, although the ICRU bladder point is easily reproducible $[8,9,10]$. This may be explained by our current findings that the $1 \mathrm{~cm}^{3}$ and $2 \mathrm{~cm}^{3}$ bladder doses are invariably higher than the ICRU bladder point dose. In our study, it was approximately 2 times higher as compared to 1.4 times as reported by others. They also suggested to treat with full bladder as the mean doses to the bladder that were lower in a distended bladder [11].

In a similar study, an evaluation of the effect of bladder filling has suggested that $5 \mathrm{cc}$ doses were higher in distended bladder. There has been no change in the dose received to the CTV [12]. However, these may not be representative, since outer wall contouring of bladder and dose to $5 \mathrm{~cm}^{3}$ may not be characteristic of bladder doses.

On a contrary, one study showed higher "hot spots" with bladder distension, and the location of the hot spots were found to be cranially located than the ICRU points in distended bladders in comparison to empty bladders [13]. Another group studied bladder filling with $50 \mathrm{cc}$, $100 \mathrm{cc}, 150 \mathrm{cc}$, and $200 \mathrm{cc}$ with sterile water, compared dosimetry to empty bladder and tried to understand the position of hot spots. The mean bladder $\mathrm{D}_{2 \mathrm{cc}}$ increased significantly by 3.5 Gy from an empty to a full bladder: 4.2 Gy to 7.7 Gy $(84.5 \%)(p=0.001)$ [14]. Our findings also show a moderate increase in mean $\mathrm{D}_{2 \mathrm{~cm}^{3}}$ bladder dose from $6.58 \pm 1.58 \mathrm{~Gy}$ in series 1 to $7.6 \pm 1.55 \mathrm{~Gy}$ in series 3 .

\section{Pattern of bladder distension and impact on other organs at risk}

We attempted to objectively evaluate the bladder distension using COV for the bladder volume. Sang Gyu et al. reported displacement of center of mass (COM) for both bladder and small bowel. The average position of the COM significantly changed in the longitudinal direction $(\mathrm{Y})$ for the small bowel (median, $-0.94 \mathrm{~cm} ; p=0.003$ ) and bladder (median, $-2.39 \mathrm{~cm} ; p<0.001$ ). The position of the $\mathrm{COM}$ of the bladder changed in the vertical direction (Z) $1.09 \mathrm{~cm}$; $(p=0.002)$. However, there was no significant volume difference in the rectum as a result of bladder distention $(p=0.412)$ [15]. The volume of the small bowel was significantly decreased in full bladder, with a median volume of $92.5 \mathrm{cc}$ (bladder filled by 4 infusion of saline; bladder filling: median 367 cc, range, 215-597). In our study, the COV and $2 \mathrm{~cm}^{3}$ volumes displacement for limited $(50 \mathrm{ml})$ and full $(100 \mathrm{ml})$ bladder filling did not differ significantly as compared to empty bladder. The overall displacement was within $5 \mathrm{~mm}$ radius. The shift of $2 \mathrm{~cm}^{3}$ volumes in superior direction was similar as reported by Sang Gyu et al. and anterior-lateral directions with larger bladder filling status. This pattern of bladder distension could be explained by the fact that vaginal packing is done after BT application, which limits the bladder filling in midline. Also, the $2 \mathrm{~cm}^{3}$ volumes displacement is minimal with bladder filling suggesting that doses measured/ delivered may be cumulative.

Mean small bowel $\mathrm{D}_{2 \mathrm{~cm}^{3}}$ dose reduced from $480 \mathrm{cGy}$ from empty bladder to $382,351,307$, and 250 cGy successively to a bladder filled with 50, 100, 150, and 200 cc sterile water [14]. Other studies also reported reduction in small bowel doses as a result of bladder filling in $[12,13]$. Our results also support the same, with an increase in bladder filling, the $2 \mathrm{~cm}^{3}$ bowel doses decrease from $4 \pm 1.69 \mathrm{~Gy}$ (empty bladder ), $3.35 \pm 2.02 \mathrm{~Gy}$ (moderate filling bladder), and $3.05 \pm 1.58 \mathrm{~Gy}$ in full bladder. This can be explained by the expansion of bladder in superior direction displacing the small bowel region around the utero-cervical region.

However in our study, there was no significant change in ICRU rectal point doses and DVH parameters for rectum and sigmoid due to bladder filling as reported in other studies $[5,8,10,16,17]$.

In recent past, there have been numerous publications on advanced brachytherapy applications including combined intracavitary-interstitial approach to address the extensive parametrial disease coverage [18]. In a recently published dosimetric study on intensity modulated radiotherapy compensation based (IMRT + ICBT) and 3D intracavitary brachytherapy, authors reported a significant increase in $\mathrm{D}_{90}, \mathrm{D}_{100}$ of HR-CTV (high risk clinical target volume), $\mathrm{D}_{90}, \mathrm{D}_{100}$, and $\mathrm{V}_{100}$ of IR-CTV dose with IMRT + ICBT plan $(p<0.05)$ in comparison to OICBT (3D optimized intracavitary brachytherapy) and CICBT (conventional 2D intracavitary brachytherapy) while the $\mathrm{D}_{2 \mathrm{cc}}$ doses to bladder, rectum, and sigmoid were significantly lower than that of CICBT and IMRT alone [19].

In advanced cervical cancer after completion of planned treatment, if complete response is not achieved, 
an application of additional sessions of brachytherapy has been conceptualized earlier. In a retrospective study after completion of planned EBRT + brachytherapy, 75 $(24 \%)$ patients received one additional ICBT and $5(7 \%)$ patients had two additional ICBT, based on post-treatment histopathological proof of residual malignant disease. No significant difference was found in grade 3 toxicity between patients who did and did not receive additional ICBT $(p=0.8)$; their series got $4 \%$ of intestinal obstruction and small bowel perforation each. So, to apply dose escalation, there is a possibility to evaluate the role of bladder filling protocol to minimize OARs dose [20]. The limitations of our study include OARs contouring uncertainties on CT imaging, inter-observer variation in contouring of OARs, limitations of the planning system to calculate small volumes especially the overlap volumes, and limited number of patients. Also this dosimetric study has been carried out on a single intracavitary BT application. The findings needs to be confirmed on a larger group of patients with preferably MRI imaging, and comprehensive evaluation of cumulative bladder dose contribution from fractionated external beam and HDR brachytherapy.

\section{Conclusions}

Bladder filling protocol with $50 \mathrm{ml}$ and $100 \mathrm{ml}$ was well tolerated and achieved a reasonably reproducible bladder volume during intracavitary brachytherapy for cervical cancer. There is no significant impact of bladder filling on bladder dose volume parameters, although larger bladder filling tend to have higher doses. Small bowel doses are lesser with higher bladder volumes. Bladder filling has no impact on rectum and sigmoid doses. The bladder $2 \mathrm{cc}$ volumes do not move significantly with increasing bladder filling.

\section{Disclosure}

Authors report no conflict of interest.

\section{References}

1. International Commission on Radiation Units and Measurements. Dose and volume specification for reporting intracavitary therapy in gynecology, Report 38. ICRU, Bethesda, MD, 1985.

2. Fellner C, Pötter R, Knocke TH et al. Comparison of radiography- and computed tomography-based treatment planning in cervix cancer in brachytherapy with specific attention to some quality assurance aspects. Radiother Oncol 2001; 58: 53-62.

3. Sun LM, Huang EY, Fat Ko S et al. Computer tomography-assisted three-dimensional technique to assess rectal and bladder wall dose in intracavitary brachytherapy for uterine cervical cancer. Radiother Oncol 2004; 71: 333-337.

4. Viswanathan AN, Dimopoulos J, Kirisits C et al. Computed tomography versus magnetic resonance imaging-based contouring in cervical cancer brachytherapy: results of a prospective trial and preliminary guidelines for standardized contours. Int J Radiat Oncol Biol Phys 2007; 68: 491-498.

5. Haie-Meder C, Pötter R, Van Limbergen E et al. Recommendations from Gynaecological (GYN) GEC-ESTRO Working Group (I): Concepts and terms in 3D image based 3D treatment planning in cervix cancer brachytherapy with emphasis on MRI assessment of GTV and CTV. Radiother Oncol 2005; 74: 235-245.

6. Jamema SV, Mahantshetty U, Tanderup K et al. Inter-application variation of dose and spatial location of D $(2 \mathrm{~cm}(3))$ volumes of OARs during MR image based cervix brachytherapy. Radiother Oncol 2013; 107: 58-62.

7. Hellebust TP, Dale E, Skjønsberg A et al. Inter fraction variations in rectum and bladder volumes and dose distributions during high dose rate brachytherapy treatment of the uterine cervix investigated by repetitive CT-examinations. Radiother Oncol 2001; 60: 273-280.

8. Orton CG, Wolf-Rosenblum S. Dose dependence of complication rates in cervix cancer radiotherapy. Int J Radiat Oncol Biol Phys 1986; 12: 37-44.

9. Crook JM, Esche BA, Chaplain G et al. Dose-volume analysis and the prevention of radiation sequelae in cervical cancer. Radiother Oncol 1987; 8: 321-332.

10. Stryker JA, Bartholomew M, Velkley DE et al. Bladder and rectal complications following radiotherapy for cervix cancer. Gynecol Oncol 1988; 29: 1-11.

11. Sun LM, Huang HY, Huang EY et al. A prospective study to assess the bladder distension effects on dosimetry in intracavitary brachytherapy of cervical cancer via computer tomography-assisted techniques. Radiother Oncol 2005; 77: 77-82.

12. Cengiz M, Gürdalli S, Selek U et al. Effect of bladder distension on dose distribution of intracavitary brachytherapy for cervical cancer: Three-dimensional computed tomography plan evaluation. Int J Radiat Oncol Biol Phys 2008; 70: 464-468.

13. Kim RY, Shen S, Lin HY et al. Effects of bladder distension on organs at risk in 3D Image-based planning of Intracavitary Brachytherapy for Cervical Cancer. Int J Radiat Oncol Biol Phys 2010; 76: 485-489.

14. Yamashita H, Nakagawa K, Okuma K et al. Correlation Between Bladder Volume and Irradiated Dose of Small Bowel in CT-based Planning of Intracavitary Brachytherapy for Cervical Cancer. Jpn J Clin Oncol 2012; 42: 302-308.

15. Ju SG, Huh SJ, Shin JS et al. Different effects of bladder distention on point A-based and 3D-conformal intracavitary brachytherapy planning for cervical cancer. J Radiat Res 2013; 54: 349-356.

16. Montana GS, Fowler W. Carcinoma of the cervix: Analysis of bladder and rectal radiation dose and complications. Int J Radiat Oncol Biol Phys 1989; 16: 95-100.

17. Sinistrero G, Sismondi P, Rumore A et al. Analysis of complications of cervix carcinoma treated by radiotherapy using the Franco-Italian glossary. Int J Radiat Oncol Biol Phys 1993; 26: 203-211.

18. Tan PW, Koh VY, Tang JI. Outpatient combined intracavitary and interstitial cervical brachytherapy: barriers and solutions to implementation of a successful programme - a single institutional experience. J Contemp Brachytherapy 2015; 7: 259-263.

19. Yin G, Wang P, Lang J et al. Dosimetric study for cervix carcinoma treatment using intensity modulated radiation therapy (IMRT) compensation based on 3D intracavitary brachytherapy technique. J Contemp Brachytherapy 2016; 8: 221-232.

20. Okuma K, Yamashit H, Kobayashi R et al. A study of highdose-rate intracavitary brachytherapy boost for curative treatment of uterine cervical cancer. J Contemp Brachytherapy 2015; 7 : 128-134. 